\title{
DESEMPENHO DA INOVAÇÃO DE PRODUTO: TESTE DE UMA ESCALA PARA APLICAÇÃO EM
}

PME's

\section{RESUMO}

A crescente pesquisa em gestão da inovação incita a necessidade de medir o desempenho proporcionado pelas variadas ações de inovação. No entanto a medição de desempenho da inovação, especialmente da inovação de produto, encontrase ainda dispersa em uma variedade de modos, seja quanto as suas entradas e saídas e/ou ainda quanto a utilizar-se dados objetivos e subjetivos. A replicação de métodos tradicionais de mensuração do desempenho da inovação de produto que não consideram os diversos resultados da inovação de produtos para a organização, agrava a questão de medida do desempenho da inovação de produto. Neste sentido, o presente estudo visa avaliar a validade e confiabilidade de uma escala multidimensional proposta por Hanachi (2015) que considera medidas de percepção, bem como o desempenho da inovação de produto como um output, para aplicação em Pequenas e Médias Empresas (PME) do setor industrial. A pesquisa é de caráter descritivo, de tipo survey e com corte transversal. A aplicação da escala de desempenho da inovação do produto foi realizada com 94 gestores de pequenas e médias indústrias do setor têxtil. Para a realização da análise empregou-se os softwares Statistica® e SPSS 2.0 onde se realizaram os testes de unidimensionalidade e confiabilidade dos constructos e posterior aplicação da análise fatorial. O resultado aponta à pertinência e aplicabilidade da escala proposta e disponibiliza uma escala válida para novos estudos que buscarem relacionar desempenho da inovação de produto com outros construtos apresentados na literatura.

Palavras-chave: Desempenho da Inovação de Produto; Escala de Mensuração; Setor Industrial; Pequenas e Médias Empresas.

\section{PRODUCT INNOVATION PERFORMANCE: PROPOSITION AND TEST OF THE ONE SCALE}

\begin{abstract}
The growing research in innovation management urges the need to measure the performance provided by the various innovation actions. However the measurement of innovation performance, especially product innovation still lies submerged in a variety of ways, which are related measurement will ace as its inputs and outputs, as well as to the objective and subjective data. The replication of traditional methods of measuring product innovation performance not consider the various results of product innovation for the organization, exacerbates the issue of measurement of product innovation performance. In this sense, the present study aims to evaluate the validity and reliability of a multidimensional scale proposed by Hanachi (2015) which considers perception measures and the performance of product innovation as an output. The descriptive research, survey type is cross-sectional. The application of product innovation performance scale was conducted with 94 managers of small and medium industries in the textile sector. To perform the analysis we used the SPSS and Statistica 2.0 software to perform the one-dimensionality and reliability testing of the constructs and subsequent application of factor analysis. The result shows the relevance and applicability of the proposed scale, by providing a valid scale for new studies that seek to relate the product innovation performance construct to other constructs allowed relationship tests, such as the approaches of structural equations.
\end{abstract}

Keywords: Product Innovation Performance; Measurement Scale; Industrial sector. 


\section{RENDIMIENTO INNOVACIÓN DE PRODUCTO: PRUEBA A ESCALA DE SOLICITUD PEQUEÑAS Y} MEDIANAS EMPRESAS

\section{RESUMEN}

La investigación cada vez mayor en la gestión de la innovación insta a la necesidad de medir el rendimiento proporcionado por las diversas acciones de innovación. Sin embargo, la medición de los resultados de la innovación, especialmente la innovación de productos aún se encuentra disperso en una variedad de maneras, ya sea como sus entradas y salidas y / o como se va a utilizar datos objetivos y subjetivos. La replicación de los métodos tradicionales de medición de resultados de la innovación de productos no considera los diversos resultados de la innovación de productos para la organización, exacerba el problema de la medición de los resultados de la innovación de productos. En este sentido, el presente estudio tiene como objetivo evaluar la validez y la fiabilidad de una escala multidimensional propuesto por Hanachi (2015), que considera que las medidas de percepción y el rendimiento de la innovación de productos como una salida para su aplicación en pequeñas y medianas empresas (PYME) el sector industrial. La investigación es descriptiva, el tipo de levantamiento y la sección transversal. La aplicación de la escala de rendimiento innovación de productos se llevó a cabo con 94 gerentes de pequeñas y medianas industrias del sector textil. Para realizar el análisis, se utilizó el software Statistica 2.0 y SPSS donde realizaron la unidimensionalidad de las pruebas y la fiabilidad de los constructos y posterior aplicación del análisis factorial. El resultado indica la relevancia y aplicabilidad de la escala propuesta y proporciona una escala válida para nuevos estudios que tratan de relacionar los resultados de innovación de productos con otras construcciones en la literatura.

Palabras clave: Actuación Innovación del Producto; Escala de Medición; Sector Industrial; Pequeñas y Medianas Empresas.

\footnotetext{
1 Doutoranda em Administração pelo do Centro de Ciências da Administração e Socioeconômicas - ESAG da Universidade do Estado de Santa Catarina - UDESC. Brasil. E-mail: nilvane_9@hotmail.com

2 Doutor em Oceanografia pela Universidade de São Paulo - USP. Professor Programa de Pós Graduação em Administração da Universidade do Vale do Itajaí - UNIVALI. Brasil. E-mail: maverdinelli@gmail.com

3 Doutor em Engenharia de Produção pela Universidade de Santa Catarina - UFSC. Professor Programa de Pós Graduação em Administração da Universidade do Vale do Itajaí - UNIVALI. Brasil. E-mail: rossetto@univali.br

${ }^{4}$ Doutor em Administração e Turismo pela Universidade do Vale do Itajaí - UNIVALI. Vice Reitor da Universidade do Contestado - UNC, Brasil. E-mail: carloseduardo@unc.br
} 


\section{INTRODUÇÃO}

Pesquisas de desempenho da inovação de produto derivaram de estudos sobre o desempenho de novos produtos e desempenho da inovação (Cooper \& Kleinschmidt, 1995; Griffin \& Page, 1995; Brown \& Eisenhardt, 1995; OECD-EUROSTAT, 1997), adquirindo destaque após a publicação de Alegre, Lapiedra \& Chiva (2006). São típicas em pesquisas relacionados à estratégia organizacional, quando se apresentam tradicionalmente como variável dependente (Keupp, Palmié \& Gassmann, 2011), e, eventualmente, como variável intermediária entre determinados processos de negócios e o desempenho geral da organização (Alegre, Lapiedra \& Chiva, 2006).

A mensuração do desempenho da inovação de produtos tornou-se um desafio, pois a utilização de diversas medidas o distanciam de um padrão (Henttonen, Ritala \& Jauhiainen, 2011; Hannachi, 2015). O desempenho da inovação de produto vem sendo mensurado tanto por intermédio de suas entradas, como a intensidade das atividades da área de P\&D (Laursen \& Salter, 2006), quanto de suas saídas, como o desempenho de produtos e processos (Terziovski, 2010). As medições envolvem ainda divergência quanto à natureza dos dados, sendo estes tanto objetivos quanto subjetivos (Alegre \& Chiva, 2008; Forfuri \& Tribó, 2009).

Dados objetivos representam um número ou percentual que atribui valor ao que se pretende medir, e em desempenho da inovação de produto podem ser medidas de rentabilidade, de desempenho da vendas, de desempenho de mercado, dentre outros (Köhler, Sofka \& Grimpe, 2012; Lin, Che \& Ting, 2012; Löfsten, 2014). No entanto o uso exclusivo de dados objetivos para mensurar desempenho da inovação de produto é apontado como inadequado mediante uma diversidade de fatores, especialmente quando aplicados em Pequenas e Médias Empresas (PME’s) (O'regan \& Ghobadian, 2004; Ulusoy \& Yegenoglu, 2005; Alegre, Lapiedra \& Chiva, 2006; Heidt, 2008; Julieti, Bakar \& Hamad, 2010; Hanachi, 2015; Chen, Wang, Nevo, Benitez-Amado \& Kou, 2015).

Neste sentido, têm sido usadas informações que refletem a percepção dos gestores e demais envolvidos nos resultados do desempenho da inovação do produtos na organização, mediante escalas subjetivas (Alegre, Lapiedra \& Chiva, 2006; Julieti, Bakar \& Ahmad, 2010; Cheng, Chang \& Li, 2013; Hanachi, 2015). As escalas subjetivas são importantes, em especial quando há falta de dados sistematizados e confiáveis sobre o desempenho da inovação de produto, e são altamente correlacionadas com medidas objetivas e informações sobre a inovação dos produtos, sendo extremamente viáveis em pesquisas com PMEs (Cheng et al., 2015).

Com o objetivo de mensurar subjetivamente o desempenho da inovação de produto foram propostas quatro escalas que, entre si, apresentam diferenças conceituais bem como dos itens de mensuração e na forma de agrupá-los (Alegre, Lapiedra \& Chiva, 2006; Julieti, Bakar \& Ahmad, 2010; Cheng, Chang \& Li, 2013; Hanachi, 2015). Anterior a estas quatro escalas, outras duas foram desenvolvidas para avaliar a inovação de produto (Li \& Atuahene-Gima, 2002) e o desempenho de novos produtos (Im et al., 2003), e que embora tenham sido replicadas para mensurar desempenho da inovação de produto, não foram desenvolvidas para esta finalidade.

Atento a questão de divergências nas escalas subjetivas de desempenho da inovação de produto, Hanachi (2015) desenvolveu pesquisa em etapa qualitativa e quantitativa para validar uma escala de desempenho da inovação de produto, com aplicação empírica em 100 industrias de biotecnologia francesas. No trabalho a terminologia adotada pela Alegre, Lapiedra \& Chiva (2006) foi descartada.

Observa-se ainda que, apesar de Julieti, Bakar e Ahmad (2010) terem validado a sua escala em Pequenas e Médias Empresas da Malásia, estas são de diversos setores, ou seja, não foi validado para um setor específico. Já a escala desenvolvida por Cheng, Chang e Li (2013) foi aplicada a um contexto específico (indústrias de alta tecnologia de Taiwan), mas foi, no entanto, elaborada a partir de uma base teórica que mensura desempenho da inovação de produto de forma objetiva, buscando mensurar a rentabilidade do produto inovador e o seu desempenho de vendas somente.

Achados na literatura apontam, ainda, a importância de pesquisas que consideram a multidimensionalidade do constructo de desempenho da inovação de produto (Henttonen, Ritala \& Jauhiainen, 2011; Dewagan \& Godse, 2014), em específico medidas que considerem o desempenho financeiro da inovação de produto (Kostopoulos et al., 2011) e o desempenho da inovação de produto para o mercado (Fóres \& Camisón, 2015). Constatando-se que a escala testada por Hanachi (2015) apresenta o maior número de dimensões propostas, bem como é aplicadas em um contexto específico de PME's.

Neste sentido, o presente estudo propõem o teste da escala proposta por Hanachi (2015) no contexto de PME's de um setor industrial brasileiro. $\mathrm{O}$ objetivo deste trabalho é, portanto, testar a validade e confiabilidade da escala subjetiva de mensuração do desempenho da inovação de produto no formato multidimensional. Baseou-se na medição proposta por Hanachi (2015), que considera o desempenho da inovação de produto quanto ao desempenho financeiro, de mercado, técnico, esperado pelo cliente e desempenho estratégico.

Pretende-se assim, contribuir com a validação de uma proposta opcional à vigente, e que considere as nuances do desempenho da inovação de produto. A escala poderá ser utilizada em estudos que necessitem de medida subjetiva, ou seja, que consideram a percepção do respondente, especialmente para aplicação em PME's do setor industrial. A validação da 
Desempenho da Inovação de Produto: Teste de uma Escala para Aplicação em PME's

escala visa a possibilitar a utilização de métodos de testes entre o desempenho da inovação de produto e outros constructos. A estrutura do trabalho apresenta inicialmente o estado da arte relativo á medidas de desempenho da inovação de produto. Segue-se o método de análise da escala e as especificações do mesmo. Apresentam-se os resultados e por fim, breve apreciação destes.

\section{DESEMPENHO DA INOVAÇÃO DE PRODUTO}

A investigação sobre o desenvolvimento de novos produtos deriva de pesquisas da década de 1970 , como exemplo os estudos SAPPHO (Rothwell, 1972; Rothwell et al., 1974) e trabalhos do Instituto de Tecnologia de Massachusetts - MIT em (1971; 1977). Especialmente a partir do ano de 1985, houve considerável acréscimo no volume de pesquisas sobre o desenvolvimento de novos produtos com foco no interesse sobre as estruturas e os processos pelos quais novos produtos são criados (Brown \& Eisenhardt, 1995).

Concomitante a busca pelos antecedentes do desenvolvimento de novos produtos, evidenciou-se a importância de verificar formas de mensurar o sucesso e o fracasso de novos produtos (Cooper \& Kleinschmidt, 1995; Griffin \& Page, 1995), e as pesquisas no plano operacional se concentraram em verificar o desempenho financeiro de novos produtos (Brown \& Eisenhardt, 1995).

Em 1997 foi lançado o Manual OSLO da Organisation for Economic Co-operation and Development - OECD, visando fornecer uma estrutura de conceitos, definições e metodologias para proporcionar as pesquisas maior compreensão do processo de inovação, buscando oferecer uma escala para a avaliação detalhada dos objetivos econômicos da inovação (OECD-EUROSTAT, 1997), e serviu de base para estudos sobre o desempenho da inovação (Yam et al., 2004; Alegre, Lapiedra \& Chiva, 2006).

Mensurações do desempenho da inovação de produto derivaram dos estudos de desempenho de novos produtos e de desempenho da inovação, e ganharam destaque principalmente após o artigo de Alegre, Lapiedra e Chiva (2006), que propõem uma escala subjetiva para avaliar especificamente o desempenho da inovação de produto, considerando a eficiência e a eficácia da inovação do produto.

A mensuração do desempenho da inovação de produtos desenvolveu-se considerando diversas medidas, tornando-se um desafio, pois não há um padrão (Henttonen, Ritala \& Jauhiainen, 2011; Hannachi, 2015). Os diferentes pontos de vista encontrados na literatura sobre o tema apontam a falta de avanço na compreensão do processo de desempenho de novos produtos (Tsai, Hsu \& Fang, 2012). Uma dificuldade relaciona-se a forma como o mesmo produto pode ser visto com diferentes graus de inovação, ou seja, um produto inovador poderia ser o primeiro de seu tipo introduzido no mercado e, portanto, também novo para a organização, ou então ser um produto que já existe no mercado, mas não em uma determinada organização (Tsai, Hsu \& Fang, 2012).

Definido como os resultados financeiros e nãofinanceiros dos esforços de inovação em produto realizado pelas organizações (Julienti, Bakar \& Hamad, 2010), desempenho da inovação de produto tem sido mensurado tanto por intermédio de seus inputs (geração de ideias, intensidade das atividades de P\&D, etc.) quanto de seus outputs (desempenho de produto e processo, desempenho financeiro, entre outros) (Garcia \& Calantone, 2002; Valadares, 2012; Grumbaum \& Stenge, 2013). Envolve ainda tanto a captação de dados objetivos (desempenho financeiro, número de patentes) quanto dados subjetivos, ditas medidas de percepção (percepção quanto aos concorrentes, percepção do desempenho) (Alegre \& Chiva 2008; Fosfuri \& Tribó, 2009).

Keupp, Palmié \& Gassmann (2011) realizaram uma revisão sistemática da literatura de 342 artigos sobre a gestão estratégica da inovação e observaram que a maioria dos estudos que analisam os resultados da inovação, a empregam como uma variável dependente, baseada principalmente em patentes, desenvolvimento de novos produtos ou desempenho financeiro. Tradicionalmente o desempenho da inovação em pesquisas de gestão tem sido utilizado como variável dependente, no entanto, pode ser considerado como uma variável intermediária entre certos processos de negócios e o desempenho geral da organização (Alegre, Lapiedra \& Chiva, 2006).

Há notável consenso na literatura sobre a necessidade de adoção da abordagem multidimensional para medir o desempenho da inovação, ou seja, que não considere somente dados financeiros e objetivos (Dewagan \& Godse, 2014). A importância de mensurar de forma multidimensional o sucesso do desenvolvimento de produtos era percebida ainda nos estudos seminais de desempenhos de novos produtos, ressaltando a não trivialidade de mensuração, e assim a importância de não basear a sua avaliação apenas uma medida (Griffin \& Page, 1993).

Cooper e Kleinschmidt (1995) apontaram como simplista o fato de utilizar apenas dados objetivos e financeiro como medida para o desempenho de novos produtos, evidenciando que novos produtos podem melhorar o desempenho de outros fatores que tem impacto na organização, como o desempenho técnico e o desempenho de mercado.

A abordagem simplista da medição de desempenho de novos produtos não considera os possíveis cenários a que a organização está sujeita, e que a medida objetiva do desempenho financeiro tornase particular ao que se mede (por exemplo, o lucro), e não ao que se pretende medir (neste caso, o desempenho da inovação de produtos) (Cooper \& Kleinschmidt, 1995).

O Manual de OSLO (OECD-EUTOSTAT, 
Desempenho da Inovação de Produto: Teste de uma Escala para Aplicação em PME's

1997) aponta ainda possíveis problemas em buscar dados sobre o desempenho financeiro, ao ressaltar que as respostas às questões sobre despesas com inovação estão entre as mais difíceis e demoradas a se obter, o número de categorias das atividades de inovação para as questões quantitativas são limitadas, incorrendo em altos custos de resposta. Julienti, Bakar e Hamad (2010) ressaltam ainda que a medição estritamente financeira pode proporcionar baixa taxa de resposta devido à relutância em compartilhar dados confidenciais.

Posterior a esses estudos, outros autores criticaram a medida unidimensional aplicada tanto ao desempenho de novos produtos, quanto ao desempenho da inovação, ou ao desempenho da inovação de produto (O'regan \& Ghobadian, 2004; Ulusoy \& Yegenoglu, 2005; Alegre, Lapiedra \& Chiva, 2006;
Heidt, 2008; Bakar \& Hamad, 2010; Hanachi, 2015).

Medidas subjetivas de desempenho da inovação de produto são altamente correlacionadas com medidas objetivas e informações sobre a inovação dos produtos lançados, e são extremamente viáveis em pesquisas com Pequenas e Médias Empresas (Cheng et al., 2015). As escalas subjetivas, no geral, solicitam aos entrevistados avaliarem a extensão na qual ocorre tal desempenho na organização e, também, na comparação entre a organização e seus principais concorrentes, sendo construídas a partir de uma escala Likert (Tsay, Hsu \& Fang, 2012). São importantes em especial quando há falta de dados sistematizados e confiáveis sobre o desempenho da inovação de produto (Cheng et al., 2015). O Quadro 1 apresenta as escalas replicadas com a finalidade de mensurar subjetivamente $o$ desempenho da inovação de produto.

Quadro 1 - Escalas desenvolvidas para mensurar desempenho da inovação e alguns estudos que as replicaram.

\begin{tabular}{|c|l|}
\hline ESCALAS DESENVOLVIDAS & \multicolumn{1}{|c|}{ REPLICAÇÕES DAS ESCALAS } \\
\hline Li \& Atuahene-Gima (2002) & $\begin{array}{l}\text { Atuahene-Gima et al., (2006); De Luca \& Atuahene-Gima (2007); } \\
\text { Molina-Castillo \& Munuera-Aleman (2009); Tsai, Hsu \& Fang (2012); } \\
\text { Chen et al. (2013); Chen et al.(2015). }\end{array}$ \\
\hline Im, Nakata, Park \& Ha (2003) & Zhang et al. (2009); Sok \& O'Cass (2015); \\
\hline Alegre, Lapiedra \& Chiva (2006) & $\begin{array}{l}\text { Alegre \& Chiva (2008); Bakar \& Ahmad (2010); Henttonen, Ritala \& } \\
\text { Jauhiainen (2001); Fernández-Mesa et al. (2013); Hannachi (2015); } \\
\text { Padilha, Gomes \& Machado (2015); }\end{array}$ \\
\hline Julienti, Bakar \& Ahmad(2010); & Replicações da escala não encontradas. \\
\hline Cheng, Chang \& Li (2013) & Replicações da escala não encontradas. \\
\hline Hannachi (2015) & Replicações da escala não encontradas. \\
\hline
\end{tabular}

Fonte: Desenvolvido pelos autores.

A primeira escala desenvolvida com o objetivo específico de mensurar o desempenho da inovação de produto foi a de Alegre, Lapiedra e Chiva (2006). A escala de Li e Atuahene-Gima (2002) foi desenvolvida com o intuito de verificar a inovação de produto e a de Im et al. (2003) com o objetivo de mensurar desempenho de novos produtos. Hanachi
(2015) observa que em cada modelo de mensuração de desempenho da inovação de produto encontram-se diferenças conceituais bem como diferenças sobre quais itens adotar e como agrupá-los. Nas escalas identificadas as dimensões utilizadas em cada pesquisa para mensurar o desempenho da inovação de produto está apresentado no Quadro 2.

Quadro 2 - Pesquisas e dimensões utilizadas para mensurar o desempenho da inovação de produto.

\begin{tabular}{|l|c|c|c|c|c|c|}
\hline \multicolumn{1}{|c|}{ Escalas } & $\begin{array}{c}\text { Li \& } \\
\text { Atuahene- } \\
\text { Gima } \\
(2002)\end{array}$ & $\begin{array}{c}\text { Im, } \\
\text { Nakata, } \\
\text { Park \& Ha } \\
(2003)\end{array}$ & $\begin{array}{c}\text { Alegre, } \\
\text { Lapiedra } \\
\text { \& Chiva } \\
(2006)\end{array}$ & $\begin{array}{c}\text { Julienti, } \\
\text { Bakar \& } \\
\text { Ahmad } \\
(2010)\end{array}$ & $\begin{array}{c}\text { Cheng, } \\
\text { Chang \& }(2013)\end{array}$ & $\begin{array}{c}\text { Hanachi } \\
(2015)\end{array}$ \\
\hline Desempenho Financeiro & $\mathrm{X}$ & $\mathrm{X}$ & $\mathrm{X}$ & $\mathrm{X}$ & $\mathrm{X}$ & $\mathrm{X}$ \\
\hline Desempenho Produto & $\mathrm{X}$ & & $\mathrm{X}$ & $\mathrm{X}$ & & \\
\hline Desempenho Processo & & & & $\mathrm{X}$ & & \\
\hline Desempenho Cliente & & $\mathrm{X}$ & & $\mathrm{X}$ & & $\mathrm{X}$ \\
\hline Desempenho Vendas & $\mathrm{X}$ & $\mathrm{X}$ & & $\mathrm{X}$ & $\mathrm{X}$ & $\mathrm{X}$ \\
\hline Desempenho Técnico & & & & $\mathrm{X}$ & & $\mathrm{X}$ \\
\hline Desempenho Geral & $\mathrm{X}$ & & & & & $\mathrm{X}$ \\
\hline Desempenho Estratégico & & & & & & $\mathrm{X}$ \\
\hline
\end{tabular}

Fonte: Desenvolvido com base nos autores. 
$\mathrm{Na}$ escala desenvolvida por Li e AtuaheneGima (2002) os autores propõem investigar o efeito da estratégia de inovação de produto sobre o desempenho de novas tecnologias na China. A escala foi desenvolvida para medir estratégia de inovação e desempenho, e as medidas foram previamente refinadas em entrevista com 23 gestores de uma população de 500 empresas de tecnologia com menos de oito anos. Foram selecionadas 300 empresas para envio do questionário, em formato Likert de 5 pontos, e obteve-se 184 respostas válidas. Procedeu-se, então, a análise fatorial onde originaram seis fatores: desempenho financeiro; desempenho da empresa ao longo dos anos; desenvolvimento de novas linhas de produtos; propriedade intelectual e patentes; auxílio governamental para implantação de tecnologia e demanda de consumo por novos produtos. Embora a escala não tenha sido desenvolvida para medir especificamente o desempenho da inovação de produto, ela foi replicada em outros estudos com essa finalidade.

A escala desenvolvida por Im et al. (2003) teve o interesse de conhecer se as empresas coreanas e japonesas estavam tendo sucesso no desenvolvimento de novos produtos, e realizaram o estudo da influência da estratégia organizacional e do processo de desenvolvimento de novos produtos no desempenho de novos produtos. Para o desenvolvimento da escala realizaram entrevistas exploratórias com gestores de produção nos dois países e, então, combinaram os resultados encontrados com a literatura, tendo como base autores como Griffin e Page (1993) e MontoyaWeiss e Calantone (1994).

O questionário focou o desempenho de novos produtos, observando os fatores aplicados no desenvolvimento de novos produtos nos 12 meses anteriores a pesquisa, e foi elaborado em modelo Likert de 5 pontos, avaliando o desempenho de novos produtos em termos de participação de mercado, vendas relacionadas, rentabilidade de novos produtos, denominadas medidas de sucesso e fracasso. Embora o questionário não tenha sido desenvolvido para avaliar o desempenho da inovação de produto, foi utilizado em ao menos dois outros estudos com essa finalidade.

Os pesquisadores Alegre, Lapiedra e Chiva (2006) foram os primeiros a desenvolver uma escala para medir especificamente desempenho da inovação de produtos, e foi replicada em no mínimo sete estudos posteriores. A escala considera a eficácia e a eficiência do desempenho da inovação de produto, sendo a eficácia da inovação os resultados econômicos da inovação de produtos, ou ainda, a importância econômica das saídas do processo de inovação, e a eficiência da inovação refere-se aos recursos consumidos para atingir estes resultados, ou seja, do próprio processo. A escala de modelo Likert de 7 pontos solicita que os respondentes avaliem seu desempenho quanto aos resultados dos principais concorrentes.
Julienti, Bakar e Ahmad (2010) buscaram verificar quais recursos contribuem com o desempenho da inovação de produto, em uma perspectiva da Visão Baseada em Recursos. Para medir desempenho da inovação de produtos adaptaram as escalas de O'Regan \& Ghobadian (2004) que mede desempenho de produto com foco no cliente, de Ulusoy (2005) que mede desempenho de produto quanto a qualidade, de Heidt (2008), que mede inovação de produto quanto á tecnologia e rentabilidade, e de Alegre, Lapiedra \& Chiva (2006) que mede a eficiência e a eficácia da inovação de produto.

Além disso, solicitaram algumas medidas objetivas, como lucro da empresa, vendas e número de funcionários. A população de pesquisa teve como base o diretório do Bank Negara Malaysia, que contém a lista de 15.150 Pequenas e Médias Empresas da Malásia em 2007, e destes, 700 pequenos e médios empreendedores foram selecionados aleatoriamente para responder ao inquérito, todos pertencentes a indústrias. Os autores concluíram que o desempenho da inovação de produto relaciona-se com uma forma específica de executar a inovação de produtos, que envolve alterações na introdução de novos produtos, técnicas e aspectos tecnológicos, resposta do mercado, qualidade dos produtos, a introdução do produto/o tempo de desenvolvimento, rentabilidade e quota de mercado.

Já Cheng, Chang e Li (2013) tinham como objetivo conhecer os potenciais antecedentes do desempenho da inovação de produtos. Realizaram o estudo em Taiwan com indústrias de alta tecnologia, de onde obtiveram 252 respondentes. A escala subjetiva foi elaborada a partir de uma base teórica que mensura desempenho da inovação de produto de forma objetiva (estudos de Danneels, 2002; Eggert et al., 2011; Evangelista \& Vezzani, 2010), e foi validada com a avaliação de quatro especialistas. Foi desenvolvida em modelo Likert de sete pontos e buscou mensurar a rentabilidade do produto inovador e o seu desempenho de vendas.

Hanachi (2015) desenvolveu um estudo analisando as escalas de mensuração de desempenho da inovação de produto desenvolvidas por Griffin (1996), Hsu \& Fang (2009), Alegre, Lapiedra \& Chiva (2006), Blindenbach et al. (2010) e Storey \& Easingwood (2009). Desenvolveu a pesquisa em etapa qualitativa e quantitativa com aplicação empírica em 100 industrias de biotecnologia francesas para testar a validade e confiabilidade da escala.

No trabalho, a terminologia adotada pela Alegre, Lapiedra \& Chiva (2006) foi descartada uma vez que (como explicado pelos entrevistados na etapa qualitativa) não leva em conta pontos importantes, tais como: melhoria da qualidade e satisfação do cliente. Ao final do estudo, Hanachi (2015) gerou dezoito itens para medir desempenho da inovação de produto classificados em cinco dimensões: desempenho financeiro, desempenho de mercado, desempenho 
técnico, o desempenho esperado pelo cliente e desempenho estratégico.

Hanachi (2015) ressalta a busca por uma escala de mensuração completa, observando as necessidades apontadas na literatura. Neste sentido, inovação de produto, ou seja, inovar, é uma decisão da organização, conforme proposta por Julienti, Bakar e Ahmad (2010) que provoca alterações na introdução de novos produtos, nas técnicas e nos aspectos tecnológicos, na resposta do mercado, na qualidade dos produtos, na rentabilidade e na maior quota do produto no mercado, e na reputação do produto, sendo estes os principais indicadores para desempenho da inovação de produto.

\section{MÉTODO DE ESTUDO}

Quanto aos meios de investigação, a pesquisa é de campo, cuja etapa de estudo ocorreu com procedimentos de coleta de dados quantitativos por intermédio da aplicação de uma survey. Os dados foram coletados em campo uma única vez, caracterizando o estudo como transversal (Hair, Babin, Money \& Samouel, 2005). Survey de amostra cujos dados são sintetizados estatisticamente são característicos de estudos descritivos (Hair et al., 2005).

Para desenvolvimento da pesquisa quantitativa constituiram-se categorias descritivas iniciais, resgatadas de conceitos a partir da plataforma teórica da investigação (Martins \& Theóphilo, 2009). Para mensurar cada variável utilizaram-se escalas, e na pesquisa optou-se por empregar escala contínua, intervalar e métrica de modelo Likert com 7 (sete) pontos (Hair et al., 2005). Para utilizar escores de um constructo para análise, deve-se certificar que as variáveis selecionadas representam e mensuram o conceito de maneira precisa (válida) e coerente (confiável) (Hair et al., 2005). Neste sentido, buscou-se amparo teórico e empírico para construção do instrumento de mensuração e validação das variáveis observando o proposto por Hair et al. (2005) quando da utilização de no mínimo três indicadores para mensurar cada variável.

O desempenho da inovação de produto foi mensurado subjetivamente, tomando como base o questionário desenvolvido por Hanachi (2015). Os pontos na escala Likert vão de 1 a 7 onde $1=$ Nunca e 7 = Muitas vezes. A escala de mensuração proposta por Hanachi (2015) apresenta cinco dimensões: desempenho financeiro da inovação de produto, desempenho de mercado, desempenho técnico, desempenho cliente e desempenho de mercado da inovação do produto, sendo que no total foi composta por dezoito indicadores.

O questionário passou pelo processo de tradução reversa e ainda foi testado com três empreendedores de pequenas empresas, para verificação da compreensão e adequação da linguagem utilizada. As PME's do setor industrial têxtil pesquisadas se encontram na região do Vale do Itajaí, cuja população contempla 246 PME’s.

O contato inicial foi realizado por telefone, quando se buscou conversar com um gerente, preferencialmente da área de produção, ou com conhecimento sobre a área. Com a confirmação da participação na pesquisa, enviou-se e-mail com o link para que pudesse ser respondido o questionário. Para enviar o questionário por e-mail, utilizou-se da ferramenta Google Forms. O resultado do contato telefônico e posterior envio de e-mail é apresentado na Tabela 1.

Tabela 1 - Resultado do contato telefônico e de e-mail.

\begin{tabular}{|l|c|}
\hline \multicolumn{1}{|c|}{ Situação - $\mathbf{1}^{\mathbf{0}}$ Contato por telefone } & $\mathbf{N}^{\mathbf{0}}$ casos \\
\hline $\mathbf{N}^{\mathbf{0}}$ telefone incorreto & 67 \\
\hline Não quis responder & 13 \\
\hline Aceitou responder & 166 \\
\hline \multicolumn{1}{|c|}{ Situação $-\mathbf{2}^{\mathbf{0}}$ Contato por e-mail: } & $\mathbf{N}^{\mathbf{0}}$ casos \\
\hline E-mail errado & 9 \\
\hline Não respondentes & 112 \\
\hline Respondentes & 45 \\
\hline
\end{tabular}

Fonte: Resultado de pesquisa.

Dos 45 respondentes, 3 foram descartados, pois eram de indústrias com menos de 20 funcionários (2) e mais de 500 funcionários (1). Concomitante ao contato telefônico realizou-se pesquisa de campo, onde se obteve 14 questionários, todos válidos, e a participação em um evento da Associação de Micro e Pequenas Empresas, onde estavam presentes 99 indústrias têxteis. Nos dois dias do evento, 47 questionários foram respondidos, sendo que destes, 38 foram considerados válidos (11 descartados por serem de indústrias com menos de 19 funcionários e um durante a análise). Desta forma, totalizaram-se 94 questionários válidos (somados aos 45 contatos via e-mail).

Para verificar se o tamanho da amostra é eficiente e adequado, considerou-se: (1) o grau de segurança, (2) nível de precisão especificado (quantidade de erro aceitável), e (3) variabilidade (homogeneidade da população) (Hair et al., 2005). Para 
fins desta pesquisa, determinou-se o grau de segurança de $95 \%$, o erro aceitável de $<0,05$. A homogeneidade da população é estimada pelo desvio padrão, e que na população de pesquisa estima-se ser homogênea, já que é representada por PME's de um mesmo setor produtivo, considerando-se assim uma distribuição normal das respostas.

Tomando como base estes dados, desenvolveuse cálculo para o tamanho mínimo de amostra considerada ideal para grandes populações (Hair et al., 2005) obtendo-se o número mínimo de 83 respondentes. Para confirmar a adequação da amostra, procedeu-se ainda o cálculo do tamanho da amostra no software Gpower®, que indicou uma amostra representativa de 88 respondentes.

Para a realização da análise empregou-se os softwares Statistica ${ }^{\circledR}$ e SPSS 2.0. A análise iniciou-se com os testes para verificar a unidimensionalidade e confiabilidade dos constructos para posterior aplicação da análise fatorial. A unidimensionalidade do constructo caracteriza-se quando todos os indicadores se ajustam a um único fator. Medidas de confiabilidade representam a adequação da análise fatorial. Para verificação de unidimensionalidade e confiabilidade dos constructos aplicaram-se as medidas de correlação, comunalidades, verificação das cargas no fator, bem como os testes de Kaiser-Meyer, de esfericidade de Bartlett e aferição da medida do Alfa de Cronbach.

Hair et al. (2009) salienta que a matriz de dados deve possuir correlações suficientes para a aplicação da análise fatorial, devendo haver assim um número substancial de correlações acima de 0,30.
Concomitante a visualização da matriz de correlação, prosseguiu-se com a identificação da estrutura latente de relações. Na pesquisa optou-se pelo método de análise de fatores comuns. A variância comum é definida como variância em uma variável que é compartilhada com todas as outras variáveis na análise (Hair et al., 2009). As comunalidades são estimativas da variância compartilhada, ou comum, entre as variáveis, e quando não puderem ser estimadas ou quando forem inválidas (valores maiores que 1 ou menores que 0) deve-se eliminar a variável de análise (Hair et al., 2009).

Já a carga fatorial é o meio de interpretar o papel que cada variável tem na definição de cada fator, ou seja, é a correlação de cada variável com o fator. Tomadas as decisões pertinentes, procedeu-se a geração de novo modelo fatorial, o qual aplicou-se os testes KMO (Kaiser, Meyer, Olkin) e de esfericidade de Bartlett, para conferir a viabilidade da análise fatorial. O teste de esfericidade de Bartlett é um teste que permite verificar a presença de correlações entre as variáveis, ou seja, fornece a probabilidade em que a matriz de correlações oferece correlações significantes em pelo menos algumas variáveis.

Verificou-se então a análise de confiabilidade de cada constructo, tendo assim o Alfa de Cronbach como uma medida diagnóstica para avaliar a consistência interna, ou seja, um coeficiente de confiabilidade que avalia a consistência da escala inteira. Os valores mínimos ideais para cada uma destas medidas estão apresentados no Quadro 3.

Quadro 3 - Valores mínimos aceitáveis para medidas de unidimensionalidade e confiabilidade

\begin{tabular}{|c|c|}
\hline MEDIDA & VALOR MíNIMO ACEITÁ VEL \\
\hline Correlação & 0,30 \\
\hline Comunalidade & 0,50 \\
\hline Carga no fator & 0,50 \\
\hline KMO & 0,60 \\
\hline Esfericidade de Bartlett & 0,05 \\
\hline Alfa de Cronbach & 0,70 \\
\hline
\end{tabular}

Fonte: Hair et al. (2006).

Após observados e ajustados os dados seguindo os critérios de medida apresentados, utilizou-se o critério de percentagem da variância com o objetivo de garantir significância prática para os fatores determinados, de forma que estes expliquem pelo menos um montante especificado da variância. Adotouse como satisfatória a medida apresentada por Hair et al. (2009) para pesquisas em ciências sociais considerando uma solução que explique no mínimo $60 \%$ da variância total.

Atendidos estes critérios, procedeu-se a análise fatorial, cujo propósito principal foi definir a estrutura subjacente em uma matriz de dados, ou seja, resumir a informação contida em diversas variáveis originais em um conjunto menor de novas dimensões compostas (fatores) com uma perda mínima de informação (Hair et al., 2014). O objetivo nesta etapa foi identificar quantos e quais fatores foram criados na análise, os indicadores e respectivos constructos.

Como critério para o número de fatores a extrair, utilizou-se o critério da raiz latente, ou método de componentes principais, onde qualquer fator individual deve explicar uma variância de ao menos uma variável. Logo, apenas os fatores que tem raízes 
Desempenho da Inovação de Produto: Teste de uma Escala para Aplicação em PME's

latentes, ou autovalores maiores que 1 são considerados significantes (Hair et al., 2009).

Observadas as cargas fatoriais e as comunalidades, procedeu-se a rotação de fatores, com a finalidade de melhorar a interpretação. Optou-se pelo método de rotação ortogonal Varimax, que se concentra na simplificação das colunas da matriz fatorial. Para interpretar os fatores, atentou-se à significância prática, ou seja, quanto maior o valor absoluto da carga fatorial, mais importante a carga na interpretação da matriz fatorial. Hair et al. (2009) ressalta que uma carga de 0,30 reflete aproximadamente $10 \%$ de explicação, e uma carga de 0,50 denota que $25 \%$ da variância é explicada pelo fator, sendo que a carga deve exceder 0,70 para que o fator explique $70 \%$ da variância.

A adoção de critérios de significância da análise fatorial seguiu as recomendações de Hair et al. (2009): a) quanto maior o tamanho da amostra, menor a carga a ser considerada significante; b) quanto maior o número de variáveis analisadas, menores as cargas a serem consideradas significantes; c) quanto maior o número de fatores, maior o tamanho das cargas em fatores posteriores a serem considerados significantes.

\section{RESULTADOS}

A apresentação dos resultados inicia-se com a análise da dimensionalidade e confiabilidade da medida de desempenho da inovação de produto. Em seguida apresenta-se o resultado da análise fatorial exploratória, cujo objetivo nesta etapa é identificar quantos e quais fatores são criados na análise e quais indicadores pertencem ao constructo.

\subsection{Análise da dimensionalidade e confiabilidade do constructo}

A Tabela 2 apresenta a correlação entre os indicadores do constructo de desempenho da inovação de produto, a comunalidade extraída em cada indicador, o valor da medida de adequação da amostra e as cargas obtidas por cada fator único extraído.

Tabela 2 - 1Correlações, cargas e índice dos indicadores das dimensões do constructo de Desempenho da Inovação de Produto

\begin{tabular}{|c|c|c|c|c|c|}
\hline \multicolumn{6}{|c|}{ Desempenho Financeiro } \\
\hline Correlação & Df1 & Df2 & Df3 & & \\
\hline Df1 & 1,000 & & & & \\
\hline Df2 & 276 & 1,000 & & & \\
\hline Df3 & 501 & 645 & 1,000 & & \\
\hline Comunalidades & ,568 & ,756 & 822 & & \\
\hline Carga Fator 1 & ,620 & ,483 & ,599 & & \\
\hline \multicolumn{6}{|c|}{ Desempenho de Mercado } \\
\hline Correlação & Dm1 & Dm2 & Dm3 & Dm4 & Dm5 \\
\hline Dm1 & 1,000 & & & & \\
\hline Dm2 & ,793 & 1,000 & & & \\
\hline Dm3 & ,638 & ,703 & 1,000 & & \\
\hline Dm4 & ,489 & ,597 & ,566 & 1,000 & \\
\hline Dm5 & ,484 &, 545 &, 582 & ,457 & 1,000 \\
\hline Comunalidades & 763 & ,841 & 739 & 635 & 688 \\
\hline Carga Fator 1 & ,703 & ,787 & ,775 & 5,565 & ,735 \\
\hline \multicolumn{6}{|c|}{ Desempenho Técnico } \\
\hline Correlação & Dt1 & Dt2 & Dt3 & & \\
\hline$\overline{\text { Dt1 }}$ & 1,000 & & & & \\
\hline$\overline{\mathrm{Dt} 2}$ & ,426 & 1,000 & & & \\
\hline$\overline{\mathrm{Dt} 3}$ & ,178 & ,473 & 1,000 & & \\
\hline Comunalidades &, 720 & ,636 & ,453 & & \\
\hline Carga Fator 1 & 0,463 & ,737 & 600 & & \\
\hline \multicolumn{6}{|c|}{ Desempenho de Cliente } \\
\hline Correlação & Dc1 & Dc2 & Dc3 & & \\
\hline
\end{tabular}


Desempenho da Inovação de Produto: Teste de uma Escala para Aplicação em PME's

\begin{tabular}{|c|c|c|c|c|c|}
\hline Dc1 & 1,000 & & & & \\
\hline Dc2 & ,433 & 1,000 & & & \\
\hline Dc3 & ,688 & ,372 & 1,000 & & \\
\hline Comunalidades & ,755 & ,578 & ,772 & & \\
\hline Carga Fator 1 & ,750 & ,573 & ,803 & & \\
\hline \multicolumn{6}{|c|}{ Desempenho Estratégico } \\
\hline Correlação & De1 & De2 & De3 & & \\
\hline De1 & 1,000 & & & & \\
\hline De2 & ,445 & 1,000 & & & \\
\hline De3 & ,454 &, 380 & 1,000 & & \\
\hline Comunalidades & ,691 & ,591 &, 507 & & \\
\hline Carga Fator 1 & ,708 & ,546 & ,648 & & \\
\hline
\end{tabular}

Fonte: Dados da pesquisa, 2015.

A variável Df2 da dimensão de Desempenho Financeiro apresentou carga no fator 1 abaixo de 0,50 $(0,483)$, portanto cogitou-se a possibilidade de retira-la da análise. Realizou-se então nova análise sem a variável e observou-se que o teste KMO ficou mais próximo do ideal com a variável Df2 (0,566 com Df2 e 0,500 sem Df2). Neste caso, optou-se por deixar a variável Df2.
A dimensão de Desempenho Técnico apresentou baixa carga no fator 1 para a variável Dt1 $(0,463)$ e baixa comunalidade para a variável Dt3 $(0,453)$, optando-se por retirar esta dimensão da análise. Após a exclusão da dimensão de Desempenho Técnico, realizou-se os testes de confiabilidade de Kaiser-Meyer-Olkin e de esfericidade de Bartlett, apresentados na Tabela 3.

Tabela 3 - Teste de adequação da escala das dimensões do constructo de Desempenho da Inovação de Produto.

\begin{tabular}{|c|c|c|}
\hline \multicolumn{3}{|c|}{ Teste de KMO e Bartlett -Desempenho Financeiro } \\
\hline \multicolumn{2}{|c|}{ Medida Kaiser-Meyer-Olkin de adequação de amostragem. } &, 566 \\
\hline \multirow[t]{3}{*}{ Teste de esfericidade de Bartlett } & Aprox. Qui-quadrado & 75,945 \\
\hline & Df & 3 \\
\hline & Sig. & 000 \\
\hline \multicolumn{3}{|c|}{ Teste de KMO e Bartlett-Desempenho de Mercado } \\
\hline \multicolumn{2}{|c|}{ Medida Kaiser-Meyer-Olkin de adequação de amostragem. } & ,838 \\
\hline \multirow[t]{3}{*}{ Teste de esfericidade de Bartlett } & Aprox. Qui-quadrado & 244,889 \\
\hline & Df & 10 \\
\hline & Sig. & ,000 \\
\hline \multicolumn{3}{|c|}{ Teste de KMO e Bartlett-Desemepnho de Cliente } \\
\hline \multicolumn{2}{|c|}{ Medida Kaiser-Meyer-Olkin de adequação de amostragem. } & ,626 \\
\hline \multirow[t]{3}{*}{ Teste de esfericidade de Bartlett } & Aprox. Qui-quadrado & 78,612 \\
\hline & Df & 3 \\
\hline & Sig. & 000 \\
\hline \multicolumn{3}{|c|}{ Teste de KMO e Bartlett -Desempenho Estratégico } \\
\hline \multicolumn{2}{|c|}{ Medida Kaiser-Meyer-Olkin de adequação de amostragem. } & ,664 \\
\hline \multirow[t]{3}{*}{ Teste de esfericidade de Bartlett } & Aprox. Qui-quadrado & 45,810 \\
\hline & Df & 3 \\
\hline & Sig. & ,000 \\
\hline
\end{tabular}

Fonte: Dados da pesquisa, 2015. 
Os testes de Bartlett apontaram significância de $0,000<0,05$ para todos os indicadores e as medidas de KMO apresenta-se inferior a 0,6 na dimensão Desempenho financeiro $(0,566)$. As demais dimensões apresentaram valores superiores a $0,6(0,838 ; 0,626$; 0,664 respectivamente). Os valores de alfas de Cronbach foram 0,726 para Desempenho financeiro, 0,873 para Desempenho de Mercado, 0,733 para
Desempenho de cliente e 0,685 para Desempenho Estratégico. Neste contexto, a fatoriabilidade da matriz foi confirmada e realizou-se o processo de extração de fatores. A Tabela 4 apresenta a variância explicada na análise fatorial exploratória dos indicadores das dimensões do constructo de Desempenho da Inovação de Produto.

Tabela 4 - Variância explicada na AFE dos indicadores das dimensões do constructo de Desempenho da Inovação de Produto

\begin{tabular}{|c|c|c|c|c|c|c|}
\hline \multicolumn{7}{|c|}{ Variância total explicada-Desempenho Financeiro } \\
\hline \multirow[b]{2}{*}{ Fator } & \multicolumn{3}{|c|}{ Valores próprios iniciais } & \multicolumn{3}{|c|}{$\begin{array}{c}\text { Somas de extração de carregamentos ao } \\
\text { quadrado }\end{array}$} \\
\hline & Total & \begin{tabular}{c|}
$\%$ de \\
variância
\end{tabular} & $\begin{array}{c}\% \\
\text { Cumulativa }\end{array}$ & Total & $\begin{array}{c}\% \\
\text { de variância } \\
\end{array}$ & $\begin{array}{c}\% \\
\text { Cumulativa } \\
\end{array}$ \\
\hline 1 & 3,438 & 65,619 & 65,619 & 3,438 & 65,619 & 65,619 \\
\hline 2 & 1,292 & 24,653 & 90,272 & & & \\
\hline 3 & ,510 & 9,728 & 100,000 & & & \\
\hline \multicolumn{7}{|c|}{ Variância total explicada - Desempenho de Mercado } \\
\hline \multirow{2}{*}{ Fator } & \multicolumn{3}{|c|}{ Valores próprios iniciais } & \multicolumn{3}{|c|}{$\begin{array}{c}\text { Somas de extração de carregamentos ao } \\
\text { quadrado }\end{array}$} \\
\hline & Total & $\begin{array}{c}\% \text { de } \\
\text { variância }\end{array}$ & $\begin{array}{c}\% \\
\text { Cumulativa }\end{array}$ & \multicolumn{3}{|c|}{\begin{tabular}{c|c|c}
\multicolumn{3}{c}{ quadrado } \\
$\%$ & $\%$ & $\%$
\end{tabular}} \\
\hline 1 & 6,936 & 66,308 & 66,308 & 6,936 & 66,308 & 66,308 \\
\hline 2 & 1,285 & 12,286 & 78,595 & & & \\
\hline 3 & 1,215 & 11,615 & 90,210 & & & \\
\hline 4 &, 660 & 6,306 & 96,516 & & & \\
\hline 5 &, 364 & 3,484 & 100,000 & & & \\
\hline \multicolumn{7}{|c|}{ Variância total explicada-Desempenho de Cliente } \\
\hline \multirow{2}{*}{ Fator } & \multicolumn{3}{|c|}{ Valores próprios iniciais } & \multicolumn{3}{|c|}{$\begin{array}{l}\text { Somas de extração de carregamentos ao } \\
\text { quadrado }\end{array}$} \\
\hline & Total & $\begin{array}{c}\% \text { de } \\
\text { variância }\end{array}$ & $\begin{array}{c}\% \\
\text { Cumulativa }\end{array}$ & Total & $\begin{array}{c}\% \\
\text { de variância } \\
\end{array}$ & $\begin{array}{c}\% \\
\text { Cumulativa } \\
\end{array}$ \\
\hline 1 & 3,711 & 65,586 & 65,586 & 3,711 & 65,586 & 65,586 \\
\hline 2 & 1,450 & 25,624 & 91,211 & & & \\
\hline 3 & ,497 & 8,789 & 100,000 & & & \\
\hline \multicolumn{7}{|c|}{ Variância total explicada - Desempenho Estratégico } \\
\hline \multirow{2}{*}{ Fator } & \multicolumn{3}{|c|}{ Valores próprios iniciais } & \multicolumn{3}{|c|}{$\begin{array}{c}\text { Somas de extração de carregamentos ao } \\
\text { quadrado }\end{array}$} \\
\hline & Total & $\begin{array}{c}\% \text { de } \\
\text { variância }\end{array}$ & $\%$ cumulativa & Total & \% de variância & $\%$ cumulativa \\
\hline 1 & 2,987 & 63,037 & 63,037 & 2,987 & 63,037 & 63,037 \\
\hline 2 & 1,008 & 21,266 & 84,303 & & & \\
\hline 3 &, 744 & 15,697 & 100,000 & & & \\
\hline
\end{tabular}

Fonte: Dados de pesquisa, 2015. 
Desempenho da Inovação de Produto: Teste de uma Escala para Aplicação em PME's

Utilizando-se o método de Kaiser para apresentar os fatores que tenham autovalor superior a 1 , constatou-se que todas as dimensões apresentaram mais de um fator com a autovalor acima de 1 , no entanto a variância explicada pelo fator 1 supera 0 mínimo recomendado de $50 \%$ em todas as dimensões $(65,619 ; \quad 66,308 ; 65,56 ; 63,037)$ demonstrando consistência na medida. Procedeu-se então a análise fatorial exploratória, explicada no tópico a seguir.

\subsection{Análise Fatorial Exploratória (AFE)}

A Tabela 5 apresenta o processo de extração de fatores, utilizando o método de componentes principais, com o critério de mostrar apenas os fatores com autovalor acima de 1.

Tabela 5 - Variância explicada da AFE dos indicadores de Desempenho da Inovação de Produto.

\begin{tabular}{|c|c|c|c|c|c|c|c|}
\hline \multirow{2}{*}{ Componente } & \multicolumn{3}{|c|}{ Valores próprios iniciais } & \multicolumn{3}{c|}{ Somas de extração de carregamentos ao } \\
quadrado
\end{tabular}

Fonte: Dados de pesquisam, 2015.

Foram extraídos quatro fatores relevantes, totalizando $65 \%$ de explicação da variância. A Tabela 6 apresenta as cargas de cada indicador sobre os três fatores extraídos bem como as comunalidades extraídas para cada indicador.

Tabela 6 - Cargas dos indicadores e comunalidades extraídas das variáveis

\begin{tabular}{|c|c|c|c|c|c|}
\hline \multirow{2}{*}{ Variáveis } & \multicolumn{3}{|c|}{ Componente } & & \\
\hline & 1 & 2 & 3 & & Comunalidade \\
\hline Df1 &, 607 &, 155 &, 066 & &, 397 \\
\hline Df2 &, 516 &, 411 &, 606 & &, 803 \\
\hline Df3 &, 587 &, 409 &, 476 & &, 739 \\
\hline Dm1 &, 734 &,- 290 &, 286 & &, 704 \\
\hline Dm2 &, 812 &,- 376 &, 134 & &, 818 \\
\hline Dm3 &, 792 &,- 256 &,- 014 & &, 694 \\
\hline Dm4 &, 607 &,- 539 &, 191 & &, 695 \\
\hline Dm5 &, 734 &,- 204 &,- 371 & &, 719 \\
\hline Dc1 &, 751 &, 025 &,- 167 & &, 593 \\
\hline Dc2 &, 546 &, 525 &,- 197 & &, 613 \\
\hline
\end{tabular}


Desempenho da Inovação de Produto: Teste de uma Escala para Aplicação em PME's

\begin{tabular}{|l|c|c|c|c|c|}
\hline Dc3 &, 808 &, 082 &,- 191 & &, 696 \\
\hline De1 &, 698 &, 350 &,- 163 & &, 636 \\
\hline De2 &, 505 &, 317 &,- 172 & &, 385 \\
\hline De3 &, 654 &, 110 &,- 309 & &, 535 \\
\hline
\end{tabular}

Fonte: Dados de pesquisa, 2015.

Os indicadores apresentaram, em sua maioria, altas cargas no fator 1 e baixas cargas ou negativas nos fatores 2 e 3, com exceção do indicador Df2 que apresentou maior carga nos fator 2. Realizou-se, então, nova análise fatorial, efetuando-se a rotação Varimax, com o objetivo de simplificar a matriz fatorial para melhor interpretação. A Tabela 7 apresenta o processo de fatoração com a rotação Varimax para os indicadores de Desempenho da Inovação de produto.

Tabela 7 - 2Processo de fatoração com a rotação Varimax para os indicadores

\begin{tabular}{|c|c|c|c|c|c|c|c|}
\hline \multirow{2}{*}{ Componente } & \multicolumn{3}{|c|}{ Valores próprios iniciais ${ }^{\mathbf{a}}$} & \multicolumn{3}{c|}{ Somas rotativas de carregamentos ao } \\
quadrado
\end{tabular}

Fonte: Dados de pesquisa, 2015.

Com a rotação, o percentual acumulado de variância explicada permanece o mesmo, ocorrendo apenas uma redistribuição da variância explicada por cada fator. A Tabela 8 apresenta o resultado da rotação Varimax, sendo omitidas as cargas fatoriais inferiores a 0,400 . 
Tabela 8 - 3Matriz de componente da rotativa.

\begin{tabular}{|c|c|c|c|}
\hline \multirow{2}{*}{ Variáveis } & \multicolumn{3}{|c|}{ Componente } \\
\hline & 1 & 2 & 3 \\
\hline Df1 & & &, 431 \\
\hline Df2 & & &, 865 \\
\hline Df3 & & &, 786 \\
\hline Dm1 & &, 752 & \\
\hline Dm2 & &, 841 & \\
\hline Dm3 & &, 716 & \\
\hline Dm4 & &, 829 & \\
\hline Dm5 & &, 578 & \\
\hline De1 &, 602 & & \\
\hline Dc2 &, 722 & & \\
\hline Dc3 &, 680 & & \\
\hline De1 &, 717 & & \\
\hline De2 &, 581 & & \\
\hline De3 &, 662 & & \\
\hline
\end{tabular}

Fonte: Dados de pesquisa, 2015.

O resultado aponta os indicadores e suas respectivas dimensões. Ressalta-se que Desempenho de cliente (Dc) e Desempenho estratégico (De) permaneceram no mesmo fator, pois durante a análise deixou-se que o software os apresentasse. Assim, retomando as questões de desempenho do cliente $\mathrm{e}$ desempenho estratégico, pode-se inferir que o desempenho estratégico é alcançado em virtude da aceitação do produto pelo cliente.

\section{DISCUSSÃO DOS RESULTADOS}

O resultado da análise fatorial confirma a multidimensionalidade do desempenho da inovação de produto proposta na teoria (Henttonen, Ritala \&
Jauhiainen, 2011; Dewagan \& Godse, 2014), quando ao final validaram-se as dimensões de desempenho financeiro, de mercado, desempenho para o cliente e desempenho estratégico como medidas de mensuração para desempenho da inovação de produto para PME's do setor industrial.

No que tange a dimensão de desempenho financeiro da inovação de produto, todos os indicadores propostos por Hanachi (2015) apresentaram ajuste satisfatório ao constructo, e relacionam-se ao incremento dos lucros e retorno sobre o investimento proporcionado pelos produtos inovadores. O Quadro 4 apresenta os indicadores validados para a dimensão de desempenho financeiro da inovação de produtos.

Quadro 4 - Indicadores validados para dimensão desempenho financeiro.

\begin{tabular}{|c|l|}
\hline DIMENSÃO & \multicolumn{1}{|c|}{ ITENS } \\
\hline \multirow{3}{*}{$\begin{array}{c}\text { Desempenho Financeiro inovação de } \\
\text { produto }\end{array}$} & $\begin{array}{l}\text { Df1: Os lucros proporcionados pelos produtos inovadores são mais } \\
\text { elevados do que o previsto aos produtos restantes. }\end{array}$ \\
\cline { 2 - 2 } & $\begin{array}{l}\text { Df2: Produtos inovadores têm alcançado os objetivos fixados em } \\
\text { termos de lucro. }\end{array}$ \\
\cline { 2 - 2 } & $\begin{array}{l}\text { Df3: Produtos inovadores têm alcançado os objetivos fixados em } \\
\text { termos de retorno sobre o investimento. }\end{array}$ \\
\hline
\end{tabular}

Fonte: Resultado análise fatorial da pesquisa. 
O desempenho financeiro, em termos de lucro, também foi utilizado na escala de Li e Atuahene-Gima (2002), que mensuraram ainda o retorno sobre ativos e a rentabilidade, solicitando que os pesquisados respondessem considerando a perspectiva da organização frente aos principais concorrentes. A perspectiva de avaliar a posição da organização frente aos concorrentes é o principal item que diferencia a escala de Li e Atuahene-Gima (2002) da proposta de Hanachi (2015), pois este último solicita aos respondentes que avaliem a percepção em termos de metas. Este aspecto pode ser considerado como positivo na escala de Hanachi (2015), já que nem sempre é possível a algumas PME's terem informações fidedignas de seus concorrentes.

Quanto a percepção de rentabilidade, Im et al. (2003) solicitam aos respondentes avaliar a rentabilidade relativa de todos os novos produtos tendo como base os últimos 12 meses de atuação da organização. A proposta de analisar a rentabilidade em caráter temporal poderia influenciar negativamente a resposta dos questionários para as PME's, visto que estas podem não ter o resultado de forma estruturada, e neste sentido a proposta de Hanachi (2015) seria a mais viável, por que utiliza a análise da rentabilidade em termos de retorno esperado sobre o investido, sendo esta também a proposta de Julienti, Bakar e Ahmad (2010).

Apesar do desempenho financeiro ser uma dimensão importante para mensurar o desempenho da inovação de produto, Keupp, Palmié e Gassmann (2011) ressaltam que ele pode ser influenciado por fontes de variação não relacionados com atividades de inovação, necessitando a análise de outros indicadores. Outro problema da mensuração do desempenho financeiro, é considerar somente o desempenho de mercado, o que poderia proporcionar problemas, pois em algum momento as organização podem ser levadas a fazer escolhas entre manter a rentabilidade ou manter a quota de mercado, e assim é importante medir estas dimensões separadamente (HANACHI, 2015).

No resultado da análise fatorial observou-se que todos os indicadores propostos para desempenho de mercado da inovação de produto apresentaram ajuste ao constructo, apontando que a separação das dimensões de desempenho financeiro e desempenho de mercado apontada nos estudos de Hanachi (2015) é factível. O Quadro 5 apresenta os indicadores validados para a dimensão de desempenho de mercado.

Quadro 5 - Indicadores validados para dimensão desempenho de mercado.

\begin{tabular}{|c|c|}
\hline DIMENSÃO & ITENS \\
\hline \multirow{5}{*}{$\begin{array}{l}\text { Desempenho Mercado inovação de } \\
\text { produto }\end{array}$} & $\begin{array}{l}\text { Dm1: Vendas de produtos inovadores são maiores do que aqueles } \\
\text { fornecidos pelo resto dos produtos. }\end{array}$ \\
\hline & $\begin{array}{l}\text { Dm2: Produtos inovadores têm alcançado os objetivos fixados em } \\
\text { termos de vendas. }\end{array}$ \\
\hline & $\begin{array}{l}\text { Dm3: Comparado com outros produtos, os produtos inovadores têm } \\
\text { alcançado resultados superiores em termos de quota de mercado. }\end{array}$ \\
\hline & $\begin{array}{l}\text { Dm4: Produtos inovadores têm alcançado os objetivos em termos de } \\
\text { quota de mercado. }\end{array}$ \\
\hline & $\begin{array}{l}\text { Dm5: Produtos inovadores têm permitido a entrada em outros } \\
\text { mercados. }\end{array}$ \\
\hline
\end{tabular}

Fonte: Resultado análise fatorial da pesquisa.

Relacionado ao desempenho de mercado, Cheng, Chang e Li (2013) buscaram verificar se produtos inovadores obtiveram vendas maiores em relação às vendas de outros produtos da empresa, assim como Hanachi (2015) que ainda buscou verificar se os novos produtos alcançaram os objetivos fixados em termos de vendas e de quota de mercado. Já Li e Atuahene-Gima (2002) e Im et al. (2003) mensuraram o desempenho de mercado mediante a verificação do retorno sobre as vendas e Julienti, Bakar e Ahmad (2010) mensuraram a evolução da quota de mercado, bem como a abertura de novos mercados.

Quanto ao desempenho do cliente da inovação de produto, ressalta-se que todos os indicadores foram validados e estão apresentados no Quadro 6. 
Desempenho da Inovação de Produto: Teste de uma Escala para Aplicação em PME's

Quadro 6 - Indicadores validados para dimensão desempenho cliente.

\begin{tabular}{|c|c|}
\hline DIMENSÃO & ITENS \\
\hline \multirow{3}{*}{$\begin{array}{l}\text { Desempenho Cliente inovação de } \\
\text { produto }\end{array}$} & $\begin{array}{l}\text { Dc1: Os clientes estão satisfeitos com o desempenho de produtos } \\
\text { inovadores. }\end{array}$ \\
\hline & $\begin{array}{l}\text { Dc2: Comparado com outros produtos, as reclamações de clientes sobre } \\
\text { produtos inovadores são menores. }\end{array}$ \\
\hline & Dc3: Produtos inovadores têm aumentado a fidelidade do cliente. \\
\hline
\end{tabular}

Fonte: Resultado análise fatorial da pesquisa.

Os estudos de Griffing e Page (1996) e Hsu e Fang (2009) evidenciram a importância de avaliar desempenho do cliente como uma dimensão específica do desempenho da inovação de produto, ao invés de incluir em uma dimensão geral. Hanachi (2015) observou esta indicação e buscou mensurar a satisfação dos clientes e a fidelidade dos clientes quanto aos produtos inovadores, o que também foi proposto por Julienti, Bakar e Ahmad (2010). Já Im et al. (2003) utilizou a percepção da participação de mercado relativa a todos os novos produtos nos últimos 12 meses de atuação da organização.

Os indicadores para a dimensão de desempenho estratégico também obtiveram validade na análise fatorial para as PME's pesquisadas. Para construção destes indicadores, Hanachi (2015) observou o proposto pelos entrevistados na etapa de pesquisa qualitativa, que apontaram a importância de analisar o desempenho da inovação de produtos no longo prazo. O Quadro 7 apresenta os indicadores validados para a dimensão de desempenho estratégico.

Quadro 7 - Indicadores validados para dimensão desempenho estratégico.

\begin{tabular}{|c|c|}
\hline DIMENSÃO & ITENS \\
\hline \multirow{3}{*}{$\begin{array}{l}\text { Desempenho Estratégico inovação de } \\
\text { produto }\end{array}$} & $\begin{array}{l}\text { De1: Produtos inovadores proporcionam à empresa uma vantagem } \\
\text { competitiva. }\end{array}$ \\
\hline & De2: Produtos inovadores têm alcançado todas as metas estabelecidas. \\
\hline & De3: Produtos inovadores têm melhorado a reputação da empresa. \\
\hline
\end{tabular}

Fonte: Resultado análise fatorial da pesquisa.

Hanachi (2015) observou o desempenho estratégico quanto à vantagem competitiva proporcionada pelos novos produtos, quanto ao alcance de metas em geral e a melhora na reputação da empresa. Li e Atuahene-Gima (2002) avaliaram o desempenho da inovação de produto no longo prazo na dimensão denominada de desempenho geral, quando buscaram a percepção relativa aos seus principais concorrentes, em função da taxa de crescimento da empresa ao longo dos anos, bem como em função da reputação global da empresa.

Ressalta-se que na rotação de fatores e dimensão de desempenho de clientes da inovação de produto e a dimensão estratégica da inovação de produtos permaneceram no mesmo fator, proporcionando inferir que o desempenho estratégico é alcançado em virtude da aceitação da inovação de produto pelo cliente.

A única dimensão proposta na escala de Hanachi (2015) que não foi validada na análise fatorial foi a do desempenho técnico, que buscou a percepção se a qualidade dos produtos inovadores é melhor do que do restante dos produtos, se os produtos inovadores são lançados nos prazos, dentro do orçado e se reduzem os danos ambientais. O Quadro 8 apresenta os indicadores da dimensão do desempenho técnico que não foram validados. 
Quadro 8 - Indicadores não validados para dimensão desempenho técnico.

\begin{tabular}{|c|l|}
\hline \multirow{2}{*}{ DIMENSÃo } & \multicolumn{1}{|c|}{ ITENS } \\
\hline \multirow{3}{*}{$\begin{array}{c}\text { Desempenho Técnico inovação de } \\
\text { produto }\end{array}$} & $\begin{array}{l}\text { Dt1: A qualidade dos produtos inovadores é melhor do que o resto dos } \\
\text { produtos. }\end{array}$ \\
\cline { 2 - 2 } & $\begin{array}{l}\text { Dt2: Produtos inovadores são lançados nos prazos. } \\
\text { Dt3: Produtos inovadores são lançados dentro dos objetivos de } \\
\text { desenvolvimento do orçamento. }\end{array}$ \\
\hline
\end{tabular}

Fonte: Resultado análise fatorial da pesquisa.

Cabe ressaltar que a escala proposta por Hanachi (2015) não incluiu a mensuração do desempenho de produto e do desempenho de processos, pois o autor considera inovações de produto e processos como antecedentes ao desempenho, ou seja, necessários para o desempenho.

Li e Atuahene-Gima (2002) mensuraram o desempenho da inovação de produto com base no desenvolvimento de novos produtos, na variedade de novas linhas de produtos e no aumento da introdução de novos produtos. Alegre, Lapiedra e Chiva (2006) mensuraram a eficiência da inovação de produto levando em consideração o custo e o tempo da inovação de projeto. Já Julienti, Bakar e Ahmad (2010) mensuraram as mudanças na introdução de novos produtos, a substituição de produtos, e a extensão da gama de produtos.

O desempenho de processo foi mensurado por Julienti, Bakar e Ahmad (2010) observando as mudanças na introdução de novos produtos quanto a qualidade do produto, o tempo de introdução do produto e seu desenvolvimento, bem como serviços de pós-venda, e a facilidade de uso e aparência. Analisando a forma como os autores mensuraram o desempenho da inovação de produto e de processo, pode-se perceber semelhanças entre ela e a dimensão de desempenho técnico proposta por Hanachi (2015), e com relação especificamente a qualidade, aos prazos de lançamento, e as metas de orçamento, ressaltando-se que a dimensão técnica não encontrou respaldo na análise fatorial para PME’s do setor industrial.

Keupp, Palmié e Gassmann (2012) apontaram o desempenho da inovação de processo como uma variável particularmente difícil de medir, especialmente por não haver consenso relacionado ao que é um processo inovador, e pela falta de compreensão da relação entre inovação, recursos e desempenho.

Por fim, ressalta-se que a terminologia adotada pela Alegre et al (2006) foi descartada da escala proposta por Hanachi (2015) uma vez que não leva em conta pontos importantes, tais como, desempenho financeiro, melhoria da qualidade e satisfação do cliente. Uma possível explicação pode ser o fato de que a escala foi criada para mensurar o desempenho da inovação de produto também como uma variável intermediária em estudos de inovação.

\section{CONSIDERAÇÕES FINAIS}

O trabalho apresentou uma escala para medir desempenho da inovação de produto que é tradicionalmente utilizada, e que busca suprir a necessidade apresentada na literatura. Neste sentido, avaliou-se a validade e confiabilidade da escala proposta por Hanachi (2015) que, ao final da análise, apresentou-se como uma alternativa pertinente, especialmente por considerar a percepção do respondente quanto ás variáveis: desempenho financeiro, desempenho de mercado, desempenho esperado pelo cliente e desempenho estratégico.

Ressalta-se que a dimensão de desempenho técnico foi excluída da escala durante os testes de unidimensionalidade. Neste sentido, a qualidade dos novos produtos, ao prazo de lançamento e objetivos de orçamento podem não medir adequadamente o desempenho técnico. Ainda na análise de unidimensionalidade, observou-se que a variável de desempenho financeiro relacionada ao alcance dos objetivos de produtos inovadores, em termos de lucro, apresentou baixa carga no fator, cogitando-se assim sua exclusão. No entanto, como observou-se que o resultado do teste de KMO ficou mais próximo do ideal com a variável, decidiu-se por sua manutenção.

Ao final dos testes restaram três fatores, apontando que os indicadores da escala demonstram multidimensionalidade, ou seja, desempenho financeiro, desempenho de mercado e desempenho esperado pelo cliente e estratégico podem ser estudados independentemente, uns dos outros, e considerados como constituintes de desempenho da inovação de produto.

Verifica-se que a satisfação dos clientes com produtos inovadores, a menor incidência de reclamações e o aumento da fidelidade dos clientes estão associados, especificamente, ao desempenho esperado pelo cliente. Aumento da vantagem competitiva, alcance de metas, e a melhora da reputação da empresa proporcionada pelos produtos inovadores relacionam-se ao desempenho estratégico.

O desempenho financeiro caracterizou-se, quando da observação, de que os lucros 
proporcionados pelos produtos inovadores são maiores, alcançando os objetivos em termos de lucros e de retorno sobre o investimento. Quanto ao desempenho de mercado, observa-se que as vendas de produtos inovadores é maior, proporcionando o alcance em termos de vendas, em termos de quotas de mercado e permitindo a entrada em outros mercados.

Este trabalho contribui, de forma importante, ao disponibilizar uma escala válida para novos estudos que buscarem relacionar o constructo de desempenho da inovação de produto a outros constructos, permitido testes de relacionamento, como os de abordagens de equações estruturais. Sugere-se ainda aplicar a escala em segmento diversos, já que a mesma foi aplicada a gestores de pequenas e médias indústrias do setor têxtil. A replicação em outros setores e em organizações de diferentes portes proporcionará a refinação da escala.

\section{REFERÊNCIAS}

Alegre, J.; Chiva, R. (2008). Assessing the impact of organizational learning capability on product innovation performance: an empirical test. Technovation, 28, pp. 315-326. Doi: http://dx.doi.org/10.1016/j.technovation.2007.09.00 3.

Alegre, J., Lapiedra, R, Chiva, R. (2006). A measurement scale for product innovation performance. European Journal of Innovation Management, 9 (4), pp. 333-346. Doi: http://dx.doi.org/10.1108/14601060610707812.

Atuahene-Gima, K.; Wei, R. (2006). The Vital Role of Problem-Solving Competence in New Product Success. Journal of Product Innovation Management, 28(1), p.81. Doi: 10.1111/j.15405885.2010.00782.x.

Bakar, L. J. A.; Ahmad, H. (2010). Assessing the relationship between firm resources and product innovation performance. Business Process Management Journal, 16, p.p. 420 - 435. Doi: http://dx.doi.org/10.1108/14637151011049430.

Blindenbach, D.F.; Ende, J.V.D. (2010). Innovation Management Practices Compared: The Example of Project-Based Firms. Journal of Product Innovation Management. 27 (5), pp. 705-724. Doi: 10.1111/j.1540-5885.2010.00746.x.

Brown, S.L.; Eisenhardt, K.M. (1995). Product development: Past research, present findings and future directions. Academy of Management Review, 20, pp. 343-378. Doi: https://ssrn.com/abstract=1506375.
Cheng, Cheng- Feng, Chang Man-Ling, Li, Chu-Shiu. (2013). Configural paths to successful product innovation. Journal of Business Research, 66, pp. 2561-2573.

Doi: http://dx.doi.org/10.1016/j.jbusres.2012.10.006.

Chen, Y.; Wang, Y.; Nevo, S.; Benitez-Amado, J.; Kou, G. (2015). IT capabilities and product innovation performance: The roles of corporate entrepreneurship and competitive intensity. Original Research Article Information \& Management, 52 (6), p.p. 643-657. Doi: http://dx.doi.org/10.1016/j.im.2015.05.003.

Cooper, R. G.; Kleinschmidt, E.J. (1995). Performance Typologies of New Product Projects. Industrial Marketing Management, 24, p.p. 439-456. Doi: 10.1016/0019-8501(95)00034-8.

Daneels, E. (2002). The dynamics of product innovation and firm competences. Strategic Management Journal, 23, p.p. 1095-1121. Doi: 10.1002/smj.275.

De Luca, I. M.; Atuahene-Gima, K. (2007). Market Knowledge Dimensions and Cross- Functional Collaboration: Examining the Different Routes to Product Innovation Performance. Journal of Marketing, 71, p.p. 95-112. Doi: http://journals.ama.org/doi/abs/10.1509/jmkg.71.1.9 5 .

Dewagan, V.; Godse, M. (2014). Towards a holistic enterprise innovation performance Measurement system. Technovation, 34, p.p. 536-545. Doi: http://dx.doi.org/10.1016/j.technovation.2014.04.00 2 .

Evangelista, R.; Vezzani, A. (2010). The economic impact of technological and organizational innovations. Research Policy, 39, p.p. 1253-1263. Doi: http://dx.doi.org/10.1016/j.respol.2010.08.004.

Forés, B.; Camisón, C. (2009). The complementary effect of internal learning capacity and absorptive capacity on performance: the mediating role of innovation capacity. International Journal Technology Management, 55 (1/2). DOI: http://dx.doi.org/10.1504/IJTM.2011.041680.

Fosfuri, A.; Tribó, J.A. (2008). Exploring the Antecedents of Potential Absorptive Capacity and its impact on Innovation Performance. Omega, 36, p.p. 173-187.

Doi: https://ssrn.com/abstract=2487636.

Garcia, R.; Calantone, R. (2002). A critical look at technological innovation typology and innovativeness terminology: a literature review. The Journal of Product Innovation Management, 19, 
p.p. $110-132$.

Doi: http://dx.doi.org/10.1016/S0737-6782(01)00132-1.

Griffin, A. (1992). Metrics for measuring new product development cycle times. Journal of Product Innovation Management 10(2), p.p. 112-125. Doi: 10.1016/0737-6782(93)90003-9.

Griffin A. (1995). PDMA research on new product development practices. Journal of Product Innovation Management, 14(6), p.p. 429-458. Doi: 10.1016/S0737-6782(97)00061-1.

Griffin, A., Page, A. L. (1993) An Interim Report on Measuring Development Success and Failure. Journal of Product Innovation Management, 10, p.p. 291-308. Doi: 10.1016/0737-6782(93)90072$\mathrm{X}$.

Grünbaum, N. N.; Stenger, M. (2013). Dynamic Capabilities: Do They Lead to Innovation Performance and Profitability? IUP Journal of Business Strategy, 10, p.p. 68-85. Doi: http://eujournal.org/index.php/esj/article/viewFile/1 $244 / 1253$.

Hair, J.F.; Babin, B.; Money, A. H.; Samouel, P. (2005). Fundamentos de métodos de pesquisa em Administração. Porto Alegre, RS: Bookman.

Hair, J. F.; Anderson, R. E.; Tatahm, R. L.; Black, W.C. (2009). Análise multivariada de dados. 6 ed. Porto Alegre: Bookman.

Hair, J. F.; Hult, G. T.; Ringle, C. M.; Sarstedt, M. (2014). A Primer on Partial Least Squares Structural Equation Modeling (PLS-SEM). Sage Publications: United Kigdom.

Hanachi, Y. (2015). Development and Validation of a Measure for Product Innovation Performance: The PIP Scale. Journal of Business Studies Quarterly, 6.

Doi: http://search.proquest.com/openview/79a6b0b890c 8d9b279d69c7cbc7d4644/1?pq-rorigsite=gscholar.

Heidt, T.V.D. (2008). Developing and Testing Model of Cooperative Interorganizational Relationships (IORS) in Product Innovation in an Australian Manufacturing Context: A Multi-stakeholder Perspective, Sourthern Cross University, Lismore. Disponível em: http://epubs.scu.edu.au/theses/70/.

Henttonen, K.; Ritala, P.; Jauhiainen, T. (2011). Exploring open Search Strategies and their perceived impact on Innovation Performance Empirical Evidence. International Journal of Innovation Management, 15, p.p. 525-541. Doi: http://dx.doi.org/10.1142/S1363919611003428.
Hsu, Y.; Fang W. (2009). Intellectual capital and new product development performance: The mediating role of organizational learning capability. Technological Forecasting and Social Change, 76 (5), p.p. 664-677. Doi: http://dx.doi.org/10.1016/j.techfore.2008.03.012.

Im, S.; Nakata, C.; Park, H.; Ha, Y.W. (2003). Determinants of Korean and Japanese new product performance: An interrelational and process view. Journal of International Marketing, 11, p.p. 81-113. Doi: https://www.jstor.org/stable/25048954.

Keupp, M. M.; Palmié, M.; Gassmann, O. (2011). The Strategic Management of Innovation: a Systematic Review and Paths for Future Research. International Journal of Management Reviews, 14, p.p. 367-390. Doi: 10.1111/j.14682370.2011.00321.x.

Köhler, C.; Sofka, W.; Grimpe, C. (2012). Selective search, sectoral patterns, and the impact on product innovation performance. Research Policy, 41(8), p.p.1344-1356.

Doi: http://dx.doi.org/10.1016/j.respol.2012.03.020.

Kostopoulos, K.; Papalexandris, A.; Papachroni, M.; Ioannou, G. (2011). Absorptive Capacity, Innovation and Financial performance. Journal Business Review, 64, p.p. 1335-1343. Doi: http://dx.doi.org/10.1016/j.jbusres.2010.12.005.

Laursen, K.; Salter, A. J. (2006). Open for innovation: the role of openness in explaining innovation performance among U.K. manufacturing firms. Strategic Management Journal, 27 (2), p.p.131150. Doi: 10.1002/smj.507. Doi: 10.1002/smj.507.

Li, Yy-Pei; Tsai, Yuh-Yuan. (2011). Knowledge Resource, Product Innovation Percormance, and the Mediating role of Knowledge Utilization Capability. Northeast Decision Sciences Institute Proccedings, p.903. Doi: http://www.nedsi.org/proc/2011/proc/p101209008. pdf.

Lin, R.; Che, R.; Ting, C. (2012). Turning knowledge management into innovation in the high-tech industry. Industrial Management \& Data Systems, 112(1), p.p.42-63. Doi: http://dx.doi.org/10.1108/02635571211193635.

Löfsten, J. (2014). Product innovation processes and the trade-off between product innovation performance and business performance. European Journal of Innovation Management, 17(1), p. p.61- 
84. Doi: http://dx.doi.org/10.1108/EJIM-04-20130034.

Molina-Castillo, F. J.; Munera- Aleman, J.R. (2009). The joint impact of quality and innovativeness on short-term new product performance. Industrial Marketing Management, 38, p.p. 984-993. Doi: http://dx.doi.org/10.1016/j.indmarman.2008.06.001.

O'Regan, N.; Ghobadian, A. (2004). The importance of capabilities for strategic direction and Performance. Management Decision, 42, p.p. 292-312. Doi: http://dx.doi.org/10.1108/00251740410518525.

OECD-EUROSTAT. (1997). The measurement of scientific and technological activities. Proposed Guidelines for Collecting and Interpreting Technological Data, OECD, Paris. Disponível em: https://www.oecd.org/sti/inno/2367580.pdf.

Padilha, C. K; Gomes, G.; Machado, D.D.P.N. (2015). Cultura de inovação e desempenho em inovação de produtos e processos: Estudo na Indústria Têxtil. SIMPOI. Disponível em: http://www.simpoi.fgvsp.br/arquivo/2015/artigos/E 2015_T00072_PCN79712.pdf.

Sok A. P; O'Cass, A. (2015). Examining the new product innovation - performance relationship: Optimizing the role of individual-level creativity and attention-to-detail. Industrial Marketing Management, 47, p.p. 156-165. Doi: http://dx.doi.org/10.1016/j.indmarman.2015.02.040.

Storey C.; Easingwood C. (1999). Types of New Product Performance: Evidence from the Consumer Financial Services Sector. Journal of Business Research, 46, p.p. 193-203. Doi: http://dx.doi.org/10.1016/S0148-2963(98)00022-8.

Terziovski, M. (2010). Innovation Practice and its Performance Implications in small and medium enterprises (smes) in the manufacturing sector: a resource-based view. Strategic Management Journal, 31, p.p. 892-902. Doi: 10.1002/smj.841.

Tsai, K.; Hsu, T. T.; Fang, W. (2012). Relinking Cross-functional Collaboration, Knowledge Integration Mechanisms, and Product InnovationPerformance: a Moderated Mediation Model. Canadian Journal of Administrative Sciences / Revue Canadienne des Sciences de Administration, 29 (1), p.p.25-39. Doi: 10.1002/cjas.192.

Ulusoy, G.; Yegenoglu, H. (2005). Innovation Performance and Competitive Strategies in the Turkish Manufacturing Industry, Sabanci University, Istanbul, 1-11. http://research.sabanciuniv.edu/7146/1/QIK2007_p aper.pdf.

Valladares, P.S.D.A. (2012). Capacidade de Inovação: Análise Estrutural e do efeito moderador da organicidade da estrutura organizacional e da gestão de projetos. Tese de Doutorado. FGV Fundação Getúlio Vargas. São Paulo. Disponível em:

http://bibliotecadigital.fgv.br/dspace/handle/10438/ 10243.

Yam, R. C. M.; Guan, J. C.; Pun, K. F.; Tang, E.P. (2004). An audit of technological innovation capabilities in chinese firms: some empirical findings in Beijing, China. Research Policy. 33, p.p. 1123-1140. Doi: http://dx.doi.org/10.1016/j.respol.2004.05.004

Zhang, J.; Hoenig, S.; Di Benedetto, A.; Lancioni, R. A.; Phatak, A. (2009). What contributes to the enhanced use of customer, competition and technology knowledge for product innovation performance?: A survey of multinational industrial companies' subsidiaries operating in China. Industrial Marketing Management, 38 (2), p.p.207218.

Doi: http://dx.doi.org/10.1016/j.indmarman.2008.12.007. 